\title{
Cytoplasmic Receptor
}

National Cancer Institute

\section{Source}

National Cancer Institute. Cytoplasmic Receptor. NCI Thesaurus. Code C17670.

Cytoplasmic Receptors are selective binding proteins that mediate the physiologic effects of specific bound regulatory molecules, such as hormones that cause a conformational and functional change in the receptor molecule. Interaction of the lig and-bound receptor with target molecules is altered, which leads to changes in cellular physiology through modification of the activity of one or more signal transduction pathways. Steroid receptors are cytoplasmic carriers that translocate to the nucleus upon ligand binding and regulate the expression of specific target genes. 